Available online at GSC Online Press Directory

GSC Biological and Pharmaceutical Sciences

e-ISSN: 2581-3250, CODEN (USA): GBPSC2

Journal homepage: https://www.gsconlinepress.com/journals/gscbps

(REVIEW ARTICLE)

\title{
A review: Brain specific delivery
}

\author{
Deepti R. Damle, Dr. Archana D. Kajale, Dr. Madhuri A. Channawar and Dr. Shilpa R. Gawande
}

Department of Pharmaceutics P. Wadhwani College of Pharmacy, Yavatmal, (M.S). 445001, India.

Publication history: Received on 28 October 2020; revised on 03 November 2020; accepted on 06 November 2020

Article DOI: https://doi.org/10.30574/gscbps.2020.13.2.0349

\begin{abstract}
The overall prevalence rate for CNS pathology has demonstrated that approximately more than one billion people are undergoing from disorders of central nervous system. The most distressing fact about delivery of drugs to the CNS is the presence of blood brain barrier that have a tendency to impair the drug distribution and denotes the major impediment for the development of CNS drugs. Neuropeptides and many drugs which are hydrophilic in nature possibly will encompass the intricacy while passing the blood brain barrier. The net amount of delivered drug (medicinal agent) and its capability to gain access to the pertinent target sites are the main considering points for CNS drug development. Brain targeted drug delivery to the brain is valuable in the diseases of brain. (Alzheimer's diseases, meningitis, brain abscess, epilepsy, multiple sclerosis, neuromylitis optica, sleeping disorders etc). Whereby high concentration can be gained with lesser side effects that occur because of release of drugs. The simplest method of targeting to brain is to obtain a therapeutic. Brain targeting systems to remain in the brain region by crossing BBB and hence significantly helps in increasing therapeutic activity. There is an increasing attraction towards brain targeting and sue to its immense application in the treatment of various CNS diseases because mostly drugs are unable to cross the BBB. This review article discuss one of the novel technology "nanotechnology" and other aspects that has been developed to target the brain and possess various clinical benefits such as reduced drug dose, less side effects, non-invasive routed, and better patient compliance.
\end{abstract}

Keywords: Brain targeting; BBB; Nanoparticles; Recent advancement; Liposomes.

\section{Introduction}

The brain specific delivery is a part of targeted drug delivery system. This targeted drug delivery system includes many aspects. Approaches, ideal characteristics, advantages, disadvantages, carriers strategies etc, i.e. in CNS targeted action can be achieved by direct administration of drugs to the CNS. [1]. BBB can impair the effect of drugs due to its absolute hindrance effect.[2].The drugs doesn't cross the BBB 100\%large molecule drugs and 98\% small molecule drugs.[3]numerous approaches of newer pharmacodynamic effects have been developed for the treatment of brain disorders[4]. Drug delivery and technology advancement like NDDS is required for the brain. [5]

The targeted drug delivery system is a special form of DOS where medicament is selectively targeted or delivered only to its site of action/abs/mol. To non- targeted tissues/cells. Methods of delivering medication to a patient in manner that increases conc. Of medication in the tissues of interest. It is mainly delivered to capillary bed of active sites/specific site of cells. To specific organ/tissue by complexion with carrier that recognizes the tablet. Its adjective is to achieve a desired pharmacological response at a selected site without undesirable interaction at other sites, thereby drug having specific site and better therapeutic index. Hence advanced technology can be used against various CNS disorders. [6]

${ }^{*}$ Corresponding author: Deepti Damle

Department of Pharmaceutics P. Wadhwani College of Pharmacy, Yavatmal.

(M.S). 445001, India.

Copyright $(2020$ Author(s) retain the copyright of this article. This article is published under the terms of the Creative Commons Attribution Liscense 4.0. 
E.g.: In cancer, chemotherapy and enzyme replacement therapy. There are many reasons for drug targeting. Also, to treat brain tumors; specifically as a part of brain specific delivery; gene therapy is also a great approach where it uses recombinant DNA to transfer a gene; and making tumor cells sensitive to a drug that cause tumor cells to die.

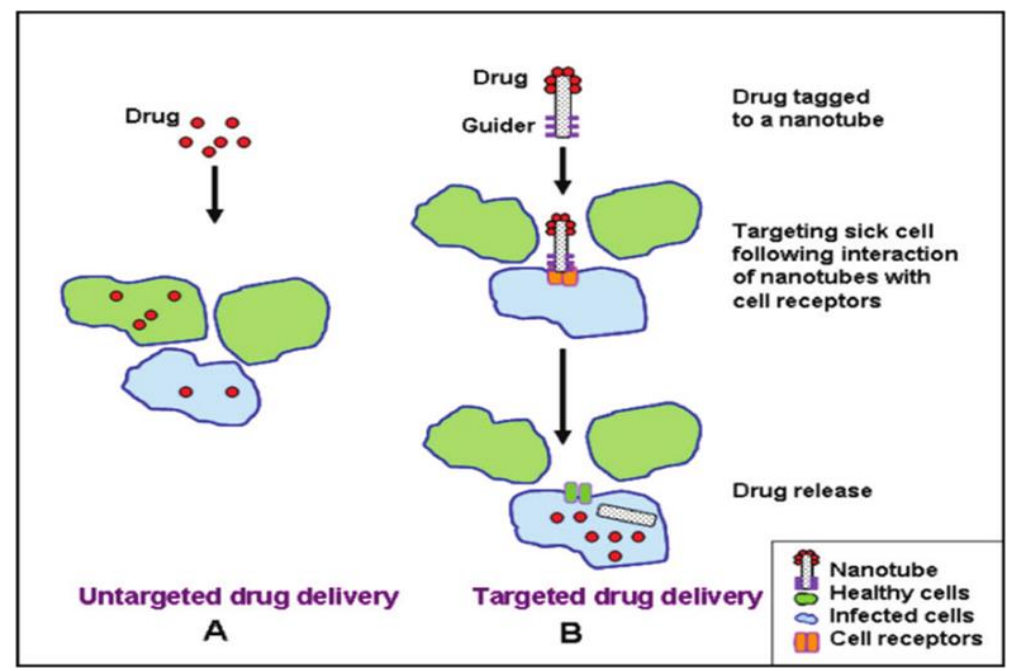

Figure 1 Drug targeting technology [7]

\section{Approaches in Brain Specific Delivery}

Specific drugs must be administered, reach the damaged cells, enter the cell and disrupt the tumor in brain. Multiple approaches have been tried; the main concern and obstacle is of the 'Blood brain barrier' in the restraining attributes as a benefit. Chemical approaches rely on chemical structure transformation of drugs to improve their unsatisfactory physicochemical properties (such as solubility or membrane penetration) and therefore change their functionalities. It is necessary to raise the systemic drug levels by substantially enhanced dose or extended administration. This often significantly increases the risk of systemic toxicity. Therefore, researchers look for more ways to transfer therapeutic agents into the CNS without the need for increasing the systemic levels of these agents. Until now, three broad categories - noninvasive, invasive, and miscellaneous techniques - have been developed to enhance drug delivery to the CNS [8]. Hence, different techniques are being used to pass the BBB and increase therapeutic effect of the drug. Mainly, we need to study the blood brain barrier. Hence, its approach is to bypass the BBB and deliver therapeutics to the brain thereby increasing drug activity has decreased toxic effects, enhancement of therapeutic efficacy and gradual release of drugs. [9]

\section{General Study of brain delivery and other aspects}

It emphasizes on drug delivery to brain and also to study the BBB. The process of passing therapeutically active mol. across the BBB; for targeting brain cells. These systems have potential to target the desired tissues and attain sustained drug release for a longer period. (Days/weeks)[10]. There are various routes of administration, as well as conjugation of drugs. With liposomes, nanoparticles are considered. Examples of drugs that can be formulation in NDDS are: Polysorbate 80-coated nanoparticles. [11] Tubocurarine [12] Doxorubicin [13].Has specific targeting along with sustained and controlled release delivery. The challenge is to deliver therapeutic agents to specific regions of brain in most brain disorder. It controls the entry of compounds into the brain barrios thereby regulates homeostasis. The capillaries lined with a layer of special endothelial cells, sealed with tight junctions. It regulates passage of molecules in and out of brain to maintain neural environment. Junctional adhesion molecules are important for trafficking of Tlymphocytes, neutrophils, and dendrite cells from the vascular compartment to the brain during immune surveillance and inflammatory responses. Adherens junctions provide strong mechanical attachments between adjacent cells and are built from cadherins and catenins. The compact network of interconnections is conferring to the endothelial layer of the BBB a transelectrical resistance $>1500 \Omega \mathrm{cm} 2$, which is the highest among all other endothelial districts. Basically, should also possess properties of hydrophilicity and lipophilicity. [14] 


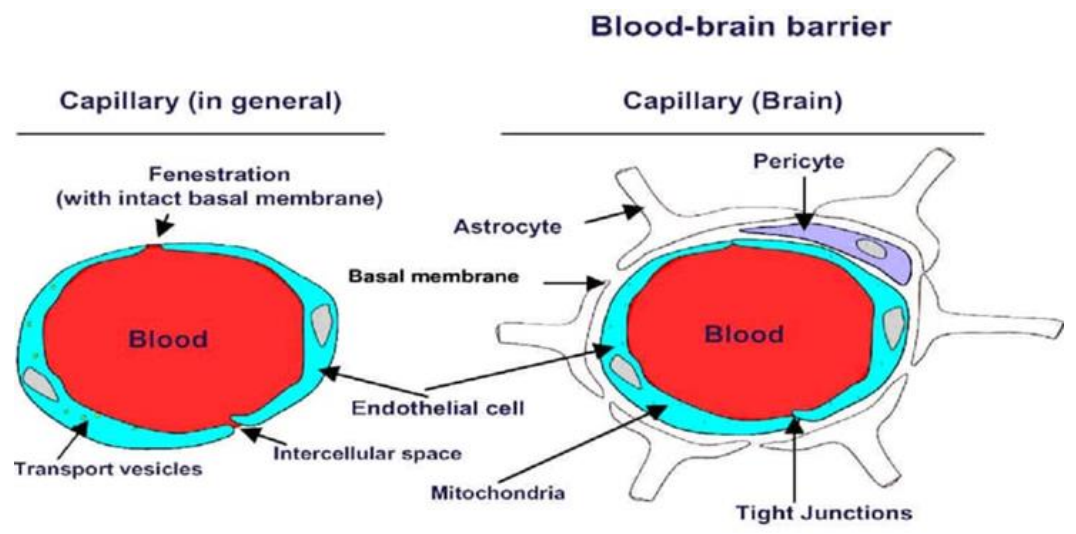

Figure 2 Capillary functions

\section{Different diseases related to BBB}

- $\quad$ Meningitis [15]

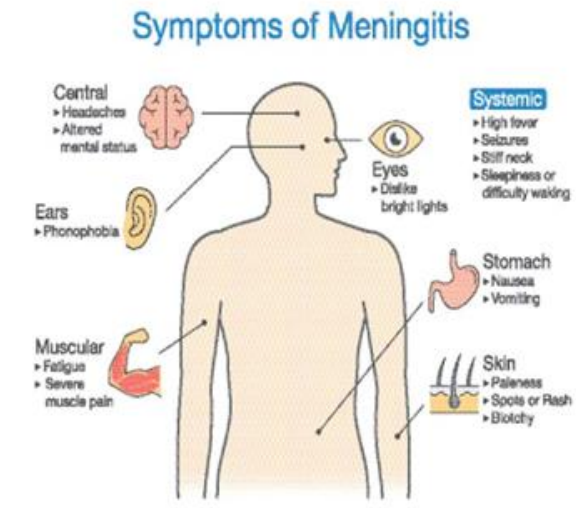

Figure3 Symptoms of Meningitis

- $\quad$ Brain Abscess [16]

- $\quad$ Epilepsy [17]

- Multiple Sclerosis [18]

- Neuromylitis Optica [19]

- $\quad$ Sleeping disorders [20]

- Alzheimer's disease [21]

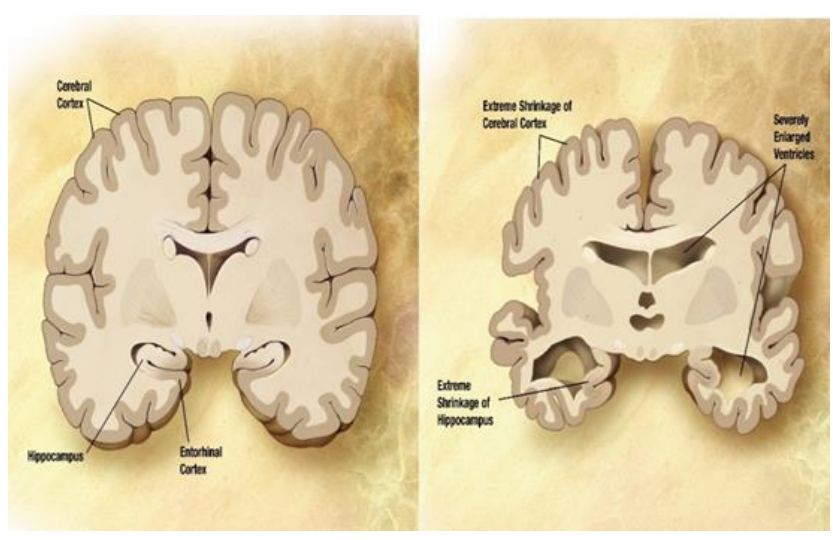

Figure 4 Alzeimer's disease [21] 


\section{Factors affecting drug delivery to Brain}

- Blood brain barrier

- Cerebro spinal fluid

- Physiochemical factors

To bypass the BBB; and deliver therapeutic effects to the brain;

The time for developing CNS drugs is normally much longer than for non-CNS drugs. Clinical trials of CNS drugs become challenging because of the complexity of the brain, side effects and the impermeable blood-brain barrier (BBB). [22]

Approaches: Invasive, pharmacological and Physiological.

- Invasive: Intra cerebro ventricular, convention enhanced Polymer/microchip Implants.

- Pharmacological: Some molecules freely enter the rainy e.g. Alcohol, Nicotine, Benzodiazepine mol size C 500 D. Penetrates the BBB.

- Physiological: Used for increasing brain delivery, transcytosis capacity. E.g. Receptor mediated transcytosis, lypophilic.

A drug must exhibit high potency and selectivity towards the biological target, as well as reach a concentration above an experimentally determined threshold within brain tissue. [23]

There are multiple functions of BBB: [24]

- As an active pump

- As a metabolic barrier

- Regulation of BBB function

- Protecting the brain from foreign substances in blood that may injure the brain

- Protects the brain from hormones and neurotransfers in the rest of the body.

\section{BBB Breakdown}

BBB breakdown occurs in neuroinflammatory diseases. [25]. NPs can transiently and reversibly open the tight junctions located at the BBB and other sites, thus, increasing their paracellular permeability. In particular, blood-brain barrier disruption therapy is an intensive, effective way of sending medication to brain tumors.

Nevertheless, it is known that tight junctions can be opened only to a limited extent; thus, only NPs smaller than about $20 \mathrm{~nm}$ can use this pathway to penetrate into the brain through the BBB.

\section{Major needs in brain specific delivery}

There is a need to target therapeutics to specific brain region / cell types. Also a need to understand BBB; transport system. Also colloidal drug carrier system vesicle, liquid crystal dispersion and size ranging from 10 to $400 \mathrm{~nm}$. [26].Need to in-vivo evaluation of brain drug pharmacokinetics. Also, a need to identify new brain drug targeting systems. Speed development and application of molecular imaging robes ad targeted contrast agents.

\section{Hurdles or Limitations of BBB}

Inadequate pharmacokinetics can limit drug therapy when the disease is located within the CNS. [27]

- $\quad$ Factors affecting drug permeation

- Time dependent plasma conc. Profile of the compound related to its distribution and elimination process.

- Binding of agent to plasma concentration and tissue and binding off rates (plasma clearance)

- Permeability of BBB to the agent

- Local cerebral blood flow

Biological process involved in Drug Targeting. 
There are widely 5 types of process involved: [28]

\subsection{Cellular uptake and processing}

- Includes pass through (frequent) various cell membrane and reach its target site. Also, the low molecular eight drugs can enter or pass through cell by simple difference.

\subsection{Endocytosis and Exocytosis}

- Oral, buccal, nasal, vaginal and rectal are internally lines with one more layer of epithelial cells

- Low molecular weight drugs from oral route are well established.

\subsection{Extravasation}

- Widely for chemotherapeutic agents.

- For in used as drug delivery to reach target site by active interaction.

- Has movement from capillary to targeted tissue.

\subsection{Lymphatic Uptake}

- As a part of targeted therapies.

- Use to develop or improve curative therapies; also to enhance mucosal immunity or oral absorption of macromolecular drugs.

\subsection{Function of RES}

- By cloaking the nano-particle with coating.

- Proportion by hydrophobic interaction to RES.

- $\quad$ Drug loaded with nano-particle; able to stay in circulation for a longer period.

Strategies of drug targeting might include both the passive and active. It gradually involves selective targeting (passive) also includes enhanced permeability retention. [29] In active targeting we can see the ligand receptor interaction also its reaching the target by virtue of blood circulation and extravasations.

For, in case of brain specific delivery; there are small colloidal particles for target sites, that includes; Alternative routes of administration, able to reach the brain bypassing the BBB (e.g., intranasal), have been actively investigated, but in the line of principle they are facing the constraints of the limited surface of adsorption of the olfactory bulb, which is minimal compared to that of the $\mathrm{BBB}$, thus, quantitatively reducing the possibility to reach the brain with relevant amounts of drugs.[30]

- Nanoparticles

- Liposomes

- Monocytes

\section{Role of Nanoparticles in Brain Specific delivery}

The nanoparticles as a targeting drug delivery system may include;

The particle size ranging from 1 to $100 \mathrm{~nm}$; having ability to target particular organ or tissue.

Having properties of hydrophobicity and lipophilicity are made up of biocompatible and biodegradable polymers. [31]

There are different types of nanoparticles; [32]

\subsection{Nanospheres}

Special particles having nanomeric dimensions and acting as a drug carrier in which drug is enclosed in polymer matrix.

\subsection{Nanocapsules}

Inner liquid core containing drug and outer surface of nanoparticles are surrounded by polymeric membrane 
Nanotechnology is a major part; the improve drug delivery to the brain can be achieved by nanotechnology; In the recent years, with the advent of nanomedicine, engineered tunable devices with the size in the order of billionth of meters have been proposed as an intriguing tool potentially able to solve the unmet problem of enhancing drug transport across the BBB, [33] a more component technology. Materials used to prepare nano-particles are polyacetates, polysaccharides, co-polymers; polysorbate coated nanoparticles, nanogels and coated nanoparticles. [34]. In the recent years, with the advent of nanomedicine, engineered tunable devices with the size in the order of billionth of meters have been proposed as an intriguing tool potentially able to solve the unmet problem of enhancing drug transport across the BBB. Among different devices, nanoparticles (NPs) technology is rapidly advancing. NPs are objects sized between 1 and $100 \mathrm{~nm}$ that work as a whole unit in terms of transport and properties.

The reasons for this expectation are most of all linked to the possibility of NPs multifunctionalization, coupled to their ability to carry drug payloads, included BBB-impermeant drugs. In particular, the rationale of using NPs for brain drug delivery is that proper surface multifunctionalization may promote at the same time either their targeting of the BBB or the enhancement of its crossing. The possibility for BBB-impermeant drugs to reach the brain, when vehicled by NPs is based upon the fact that their crossing of the barrier will depend completely on the physicochemical and biomimetic features of the NPs vehicle and will not depend anymore on the chemical structure of the drug, which is hindered inside the NPs.

What makes NPs even more attractive for medical applications is the possibility of conferring on them features such as high chemical and biological stability, feasibility of incorporating both hydrophilic and hydrophobic pharmaceuticals, and the ability to be administered by a variety of routes[35] (including oral, inhalational, and parenteral). Moreover, NPs can be functionalized by covalent conjugation to various ligands (such as antibodies, proteins) to target specific tissues. The large surface-area-to-volume ratio of NPs permits multiple copies of a ligand to be attached and to dramatically increase their binding affinity via the multivalent functionalization. [36]

When designing NPs for clinical applications, it should be remembered that their systemic administration generates important modifications. In particular, the nonspecific interaction between the shell of NPs and many classes of proteins circulating in the bloodstream leads to the adsorption of opsonins on their surface, forming the so-called "corona." These proteins substantially change the bare material properties determining the removal of NPs from circulation by the reticuloendothelial system, mainly located in spleen and liver. The most common approaches used for escaping RES are to formulate the particles with neutral surface charge, to coat their surface with different hydrophilic surfactants, such as polysorbates and polyethylene glycol (PEG), and to use small size nanoparticles (e.g., $<80 \mathrm{~nm}$ )[37]. NPs with these features, called "stealth," are able to avoid the reticuloendothelial system, to display long circulation time and stability in blood, and may be functionalized to successfully target and cross the BBB.[38] Finally, NPs should be nontoxic either for cells in the bloodstream or for healthy bystanding cells and should be biodegradable and biocompatible, noninflammatory and non-immunogenic.

The liposomes and nanocytes also play a very important role in brain specific drug delivery system.

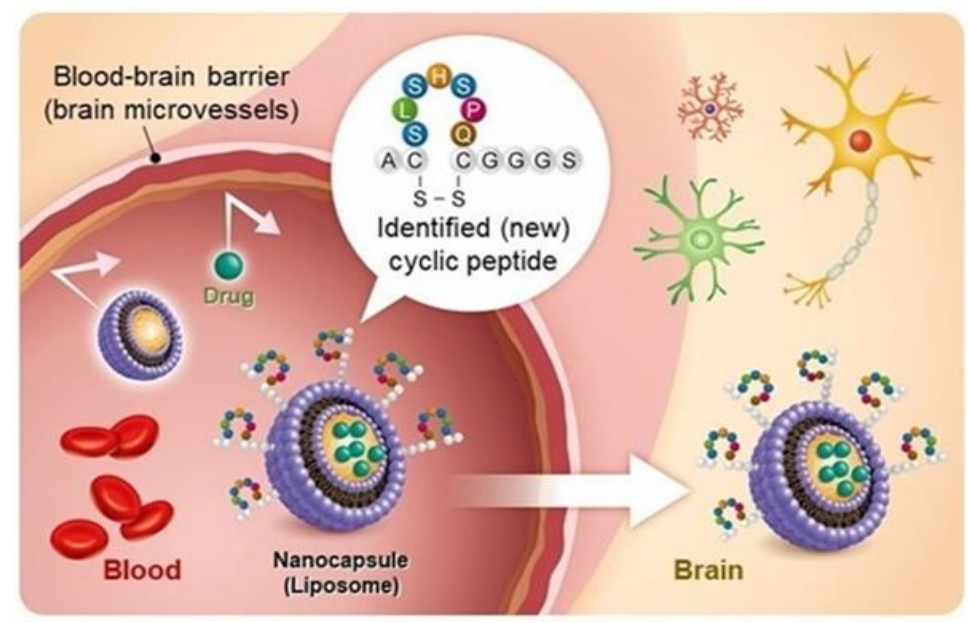

Figure 5 Liposomes and Nanoparticles for treatment in DDS 


\section{Liposomes}

They consist of a direct delivery of drug into the brain, chemical modification of drug making them permeable and using specific receptors expressed in the BBB surface to transport the drug onto the brain. Liposomes are the first generation of nanoparticulate drug delivery systems and are constituted by one or more vesicular bilayers (lamellae) composed of amphiphilic lipids, delimiting an internal aqueous compartment. [39] Usually, the liposomal lipid bilayer is composed of biocompatible and biodegradable lipids, present in biological membranes. Common liposome constituents are sphingomyelin, phosphatidylcholine, and glycerophospholipids. Cholesterol, an important component of cell membranes, is frequently included in liposome formulations because it decreases the bilayer permeability and increases the stability of the liposome in vivo.

Like; a small portion of drug will be likely to reach the innermost olfactory region; where they can be transported to the brain by three different routes; i) Intracellular ii) Extracellular and iii) Transcellular transport.

Liposomes are classified on the base of their size and the number of lamellae as follows (i) small unilamellar vesicles (SUV) with a size up to $100 \mathrm{~nm}$ and one bilayer, (ii) large unilamellar vesicles (LUV) with a size $>100 \mathrm{~nm}$ and one bilayer, and (iii) multilamellar vesicles (MLV) that can reach a size of several $\mu \mathrm{m}$ and made of many concentric lipid bilayers.

Liposomes have been largely utilized for brain drug delivery [40], for the treatment of cerebral ischemia, for delivery of peptides, and brain tumours.

Cationic Liposomes: Cationic liposomes containing positively charged lipids have been developed and initially used as transfection vehicles, to deliver genetic material (e.g., DNA) into the cell, avoiding the lysosomal digestion. The most commonly utilized cationic lipid is 1,2-dioleoyl-3-trimethylammonium-propane (DOTAP), mixed with dioleoylphosphatidylethanolamine (DOPE). The cholesterol also increases the levels of transfection and can potentially reduce the destabilization of the liposomes in the presence of serum.

The interactions between cationic lipids and nucleic acids lead to the formation of structures, which are called "lipoplexes" [41]. Lipoplexes are typically formed by direct mixing between cationic liposomes and DNA solutions. Positively charged liposomes bind to negatively charged phosphate molecules on the DNA backbone through electrostatic interactions that are embedded and are shielded from the environment. Generally, complexes are formed with a slight excess positive charge to permit them to interact with the negatively charged cell surface. The cationic liposomes used are typically small before adding to DNA; however, complexes formed with DNA exhibit diameters that range from as small as $200 \mathrm{~nm}$ to structures as large as $2 \mu \mathrm{m}$. Unlike liposomes, cationic liposomes undergo adsorptivemediated endocytosis and internalization in endosomes. Upon acidification at pH 5 to 6, DOPE fuses and destabilizes the endosomal membrane, releasing its contents to the cytosol. Therefore, drugs could be vehicled into endothelial cells, similarly to DNA, enhancing their crossing of the barrier and reaching neurons. As a proof of this possibility, transfection of neuronal SH-SY5Y cells was achieved with the lipoplexes at a degree much higher than the degree obtained with the widely and commonly utilized transfectant Lipofectamine; cationic liposomes carrying a photo reactive drug resulted in a laser-stimulated cytotoxic effect on glioblastoma cells and showed the ability to improve brain drug delivery of paclitaxel (a mitotic inhibitor used in cancer chemotherapy) in rodents in vivo.

\section{Monocytes in brain drug delivery}

A typical feature of neuroinflammation response; is the recruitment of leucocytes; (mainly monocytes and neutrophils), [42] to the lesion in brain under pathological condition; to exploit these inflammatory cells as targeted delivery systems. The ability of nanocytes and monocytes derived macrophages (MDM) to travel towards chemotactic gradient, traverse tissue barriers and accumulate precisely at diseased sites; makes them attractive candidates as drug carriers and therapeutic gene delivery vehicles targeting the brain where treatments are often hampered.

\section{Challenges of CNS drug development}

CNS disorders treatable with small drug molecular therapy [43]

- Depression

- Schizophrenia

- Chronic pain

- Epilepsy 
CNS disorders that is refractory to small drug molecular therapy [44]

- Multiple sclerosis, Brain cancer, trauma

- Alzheimer's, Parkinson's

- Blindness

- Cerebro vascular disease

\section{Hurdles/Challenges in Brain Specific Delivery}

- The difficulty of delivering therapeutic agents, to specific regions of the brain presents a major challenge to treatment of most brain disorders.[45]

- $\quad$ There are number of barriers that inhibit drug delivery to CNS.

- Drug delivery to neoplastic cells in a solid tumor is compromised by a heterogeneous distribution of microvasculature through the tumor interstitial, which leads to spatially inconsistent drug delivery.

- The capillaries of the brain have evolved to constrain the movement of molecules and cells between blood and brain, providing a natural defence against circulating toxic, or infections agents.

- Even if the use of engineered NPs represents one of the main hopes for innovative pharmacological strategies in neurology, it is important to mention that the BBB represents a mechanism of defence of CNS against potentially neurotoxic molecules and structures, NPs included. In vivo and clinical data evaluating the toxic effects of NPs on neural cells are still scarce, and it is still difficult to extrapolate the results obtained on in vitro models to the actual situation in vivo, given that the application of NPs to the CNS is at a nascent stage.

\section{Deaths}

- It is estimated that 8,020 adults; (10,190 men and 7,830 women) will die from primary cancerous brain and CNS tumors this year. The 5 year survival rate tells you what percent of people live at least 5 years after the tumor is found. Percent as much many out of 100. [46]

\section{Speculative Uses}

- It is the BBB that acts and effectively protects the brain from infections.[47]

- Tumors and infections do occur and are often serious and difficult to treat. Antibodies are too large to cross the blood brain barrier; only certain are able to pass, hence colloidal particles are used.

- $\quad$ Some substance, like fat soluble substances can pass. E.g. Alcohol, nicotine and caffeine.

- The approach on brain delivery, utilizes knowledge to link drugs with amino acids that actively cross the BBB.

- As per the strategies; hydrophilic molecules such as proteins and peptides enter the brain through specific and saturable receptor mediated transport mechanism; as glucose.

- These luminal transporters are expressed at the luminal and albuminal EC membranes.

\section{Material and methods}

- Dual targeting from/for brain specific delivery; synthesis and biological evaluation.

- Ibuprofen is one of the most potential NSAIDs and plays an important role in treatment of neurogenerative diseases.

- (Though; it has poor brain penetration and has large side effects. It could be released from prepared prodrugs.)

- Naproxen and indomethacin have been utilized.

- In-vitro prodrugs stability has been investigated.

- $\quad$ LAT1- utilizing prodrugs of several different compounds.

- In case of Alzheimer's disease, astrocytes achieved the results.

- Ketoprofen and anti-oxidant ferulic acid displayed improved cellular uptake.

There are other anti-inflammatory and neurotransmitter drugs for easy penetration into the BBB, which is used for the treatment of neural disorders. Mostly small size particulate substances are used as stated earlier (Nanoparticles, other colloidal particles etc.).[48] 


\section{Mechanism}

Basically, mechanisms include all the aspects studied earlier. Mainly focus on the targeted drug delivery systems. Overall, it specifically revolves around the BBB passing because BBB is the main barrier from protecting against foreign bodies. Hence, only certain materials can pass through.

Mechanisms depend on the materials and methods along with the strategies and active and passive targeting. Many different approaches where in the drug has to pass BBB. The transport is through carrier mediated transport system and receptor mediated transcytosis.

The permeability is also a factor affecting and also the local cerebral blood flow. Plasma clearance can be also seen.

Some approaches in mechanism might involve the invasive, pharmacological and physiological materials (Implants, Benzodiazepine, carrier mediated etc. [49]

The brain specific drug delivery is a part of targeted drug delivery system. Mainly it's important because other systems might have easy penetration of drug, but in case of brain, due to barriers it is hard to penetrate and pass through blood brain barrier as brain is the most protected part of body.

We can also state that the nanoparticles are boon to the brain target delivery; hence/since there are remarkable results in terms of drug penetration. Strategies at increasing brain uptake of drugs that are substrates for specific efflux mechanisms, etc.

These are the theoretical parts of discussion. Practically it is not very easy but due to new, novel technology and drug development; it is made possible. Synthetic and biological processes are involved with its synthesis. In dual targeting; as a result more than $98 \%$ of small molecule drugs and nanoparticles penetrate, and give powerful results.

Photodynamic therapy, Photofrin along with iron oxide nanoparticles used to target tumor cells. Nanochip for enhancement of nanoparticles release.

\section{Future Prospects}

To develop several systematically effective neuropharmaceuticals; effective after systemic administration.

E.g.: If a given peptide/protein has a transport system; at the luminal site of BBB; could be modified to use the existing transport mechanism. [50]

\section{Recent Advances}

Recent Advances includes

- 20-20 nm, diameters of probes encapsulated by biologically localized embedding in brain cancer targeting.

- Chimeric peptide technology.

- Lipobridge Technology.

- Peptide Radio pharmaceuticals.

- Trojan horses coated with sugar layer, another modern approach containing magnetized, iron containing nanoparticles.[51]

\section{Conclusion}

Now-a-days, many researchers are attracted towards brain targeting; due to its immense application in the treatment of various CNS diseases because mostly drugs are unable to cross the Blood brain barrier. This short review article discuss the one of the novel technology "nanotechnology" and other aspects; that has been developed to target the brain and possess various clinical benefits such as reduced drug dose, less side effects, non-invasive routes, and letter patient compliance. Neuropeptides and many drugs which are hydrophilic in nature possibly will encompass the intricacy while passing the blood brain barrier. Brain targeting systems to remain in the brain region by crossing BBB and hence significantly helps in increasing therapeutic activity. There is an increasing attraction towards brain targeting and sue 
to its immense application in the treatment of various CNS diseases because mostly drugs are unable to cross the BBB. This review article discuss one of the novel technology "nanotechnology" and other aspects that has been developed to target the brain and possess various clinical benefits such as reduced drug dose, less side effects, non-invasive routed, and better patient compliance.

\section{Compliance with ethical standards}

\section{Acknowledgments}

I would like to express my deep gratitude to my guides Dr. Archana D. Kajale, Dr. Madhuri A. Channawar and Dr. Shilpa R. Gawande mam for their valuable support and guidance and constructive suggestions during this article. Their willingness to give the time and generously helping is appreciated. Finally, I wish to thank my parents for their encouragement and support throughout.

\section{Disclosure of conflict of interest}

No conflict of interest.

\section{References}

[1] A Misra, C Ganesh, Shahiwala, SP Shah.Drug delivery to current nervous system: a review J Pharm Pharm Sci. 2003; 6: 252-73.

[2] AH Schinkel, E Wagennar, C Mol, L van Deemter.P-glycoprotein in the blood brain barrier of mice influences the brain penetration and pharmacological activity of many drugs Journal of Clinical Investigation. 1996; $97: 2517$.

[3] WM Pardridge.Blood-brain barrier drug targeting: the future of brain drug development Molecular interventions. 2003; 3: 90.

[4] W Loscher, H Potschka.Blood-brain barrier active efflux transporters: ATP binding cassette gene family NeuroRx. 2005; 2: 86-98.

[5] MA Moses, H Brem, R Langer.Advancing the field of drug delivery: taking aim at cancer Cancer cell. 2003; 4: 337341.

[6] MI Alam, S Beg, A Samad, S Baboota, K Kohli, J Ali, M Akbar, Strategy for effective brain drug delivery European journal of pharmaceutical sciences. 2010; 40: 385-403.

[7] DR Groothuis.The blood-brain and blood-tumor barriers: a review of strategies for increasing drug delivery Neuro-oncology. 2000; 2: 45-59.

[8] Tsuji A. Small molecular drug transfer across the blood-brain barrier via carrier-mediated transport systems. NeuroRx. 2005; 2:54-62.

[9] R Singh JW Lillard.Nanoparticle-based targeted drug delivery Experimental and molecular pathology. 2009; 86: 215-223.

[10] S Acharya, SK Sahoo.PLGA nanoparticles containing various anticancer agents and tumour delivery by EPR effec Advanced drug delivery reviews. 2011; 63: 170-183.

[11] JM Koziara, PR Lockman, DD Allen, RJ Mumper In situ blood-brain barrier transport of nanoparticles Pharmaceutical research. 2003; 20: 1772-1778.

[12] A Kumari, SK Yadav, SC Yadav. Biodegradable polymeric nanoparticles based drug delivery systems Colloids and Surfaces B: Biointerfaces.2010; 75: 1-18.

[13] KA Janes, MP Fresneau, A Marazuela, A Fabra, MAJ Alonso. Chitosan nanoparticles as delivery systems for doxorubicin Journal of controlled Release. 2001; 73: 255-267.

[14] T Neuberger, B Schöpf, H Hofmann, M Hofmann, B. Von Rechenberg.Superparamagnetic nanoparticles for biomedical applications: possibilities and limitations of a new drug delivery system Journal of Magnetism and Magnetic Materials. 2005; 293:483-496.

[15] MF Heyderman, RS Michael BD et al. The UK joint specialist socities guideline on the diagnosis and management of acute meningitis and meningococcal sepsis in immunocompetent adults. J Infect. 2016; 72:405-38. 
[16] D Muzumdar Central nervous system infections and the neurosurgeon: A perspective. Int J Surg. 2011; 9:113-6.

[17] A Paoli, M Canato, L Toniolo, AM Bargossi, M Neri, M Mediati, D Alesso, G Sanna, KA Grimaldi, AL Fazzari, A Bianco. The ketogenetic diet: an underappreciated therapeutic option? La Clinican Terapeutica. 2011,2009;162, e-145e153.

[18] MM Abdel Fatah, SY Alokbi, Ramadan, SKholoud, DA Mohamed, SE Mohammed. 2009.

[19] Jarius S, Wildemann B. The history of neuromylitis optica. J Neuroinflammation. 2013; 10:8.

[20] Understanding Sleep: Brain Basics. Office of Communications and Public Liaison, National Institute of Neurological Disorders and Stroke, Bethesd, Md.

[21] E Bossy-Wetzel, R Schwarzenbacher, SA Lipton. Molecular pathways to neurodegeneration, Nature Medicine. 2004; 10: 2-9.

[22] Lingineni K, Belekar V, Tangadpalliwar SR, et al. The role of multidrug resistance protein (MRP-1) as an active efflux transporter on blood-brain barrier (BBB) permeability, Mol Divers. 2017; 21:355-65.

[23] Medicinal chemical properties of successful central nervous system drugs. Pajouhesh H, Lenz GRNeuroRx. 2 Oct. 2005; 4:541-53.

[24] Abbott NJ, Patabendige, AA Dolman, DE Yusof, SR Begley DJ.Structure and Function of the blood-brain barrier. Neurobiol Dis. 2009; 37:13-25.

[25] Abbott NJ, Khan EU, Rollinson CM, Reichel A, Janigro D, Dombrowski SM, Dobbie MS, Begley DJ, Drug resistance. BBB breakdown. 2002; 243:38-47.

[26] C Muller-Goymann. Physiochemical characterization of colloidal drug delivery systems such as reverse micelles vesicles liquid crystals and nanoparticles for topical administration.

[27] Tam SJ, Richmond DL, Kaminker JS, Modrusan Z, Martin-McNulty B, Cao TC, Weimer RM, Carano RA, Bruggen N, Watts RJ. Death receptors DR6 and TROY regulate brain vascular development. Dev Cell. 2012; 22:403-17.

[28] Modern Pharmaceutics (Gilbert S.Banker, 4th edition) Targeted drug delivery system, Vyas and Khar Biopharmaceutics and Pharmakokinetics; Bramhankar.

[29] S.C Rastogi, K.Sambulingam (Anatomy and Physiology); Modern Pharmaceutics.

[30] A Minn, S Leclerc, JM Heydel et al.Drug transport into the mammalian brain: the nasal pathway and its specific metabolic barrierJournal of Drug Targeting, 2002;10(4): 285r-296r.

[31] M Young, JD Hoheisel, T Efferth.Therapeutic and diagnostic applications of nanoparticles Current Drug Targets. 2011; 12: 357-365.

[32] L Braydich Stolle, S Hussain, JJ Schlager, MC Hoffman. In-vitro cytotoxicity of nanoparticles in mammalian germline stem cells. Toxicological sciences. 2005; 88: 412-419.

[33] FRe, M Gregori, M Masserini. Nanotechnology for neurodegenerative disorders Nanomedicine NBM. 2012; 8(1): S51-S58.

[34] C Pardeshi, P Rajput, V Belgamwar et al.Solid lipid based nanocarriers: an overview Acta Pharmaceutica. 2012; 62(4): 433-472.

[35] KC Petkar, SS Chavhan, S Agatonovik-Kustrin, KK Sawant.Nanostructured materials in drug and gene delivery: a review of the state of the art Critical Reviews in Therapeutic Drug Carrier Systems. 2011; 28(2): 101-164.

[36] X Montet, M Funovics, K Montet-Abou, R Weissleder, L Josephson.Multivalent effects of RGD peptides obtained by nanoparticles display Journal of Medicinal Chemistry. 2006; 49(20):6087-6093.

[37] JM Provenzale GA Silva.Uses of nanoparticles for central nervous system imaging and therapy American Journal of Neuroradiology. 2009; 30(7): 1293-1301.

[38] R Gabathuler.Approaches to transport therapeutic drugs across the blood-brain barrier to treat brain diseases Neurobiology of Disease. 2010; 37(1): 48-57.

[39] M Budai M Szógyi.Liposomes as drug carrier systems. Preparation, classification and therapeutical advantages of liposomes Acta Pharmaceutica Hungarica. 2001; 71(1): 114-118.

[40] F Lai, AM Fadda, C. Sinico.Liposomes for brain delivery Expert Opinion on Drug Delivery. 2013. 
[41] F Artzner, R Zantl, JO Rädler.Lipid-DNA and lipid-polyelectrolyte mesophases: structure and exchange kineticsCellular and Molecular Biology. 2000; 46(5): 967-978.

[42] Nanomedicine. Feb 2015; 11(2):391-400.

[43] K Honjo, SE Black, NP Verhoeff.Alzheimer's disease, cerebrovascular disease, and the $\beta$-amyloid cascade Canadian Journal of Neurological Sciences. 2012; 39(6): 712-728.

[44] JR Kanwar, B Sriramoju, RK Kanwar.Neurological disorders and therapeutics targeted to surmount the bloodbrain barrier International Journal of Nanomedicine. 2012; 7: 3259-3278.

[45] Pehlivan SB. Nanotechnology-based drug delivery systems for targeting, imaging and diagnosis of neurodegenerative diseases. Pharm Res. 2013; 30:2499-511.

[46] Statistics adapted from the American Cancer Society's publication, Cancer Facts \& Figures, the ACS website, the CBTRUS Statistical Report: Primary Brain and Other Central Nervous System Tumors Diagnosed in the United States in, and the National Cancer Institute website. 2012-2016, January 2020.

[47] MA Petty EH Lo.Junctional complexes of the blood-brain barrier: permeability changes in neuroinflammationProgress in Neurobiology. 2002; 68(5): 311-323.

[48] Fan W, Wu Y, Li XK, et al. Design, synthesis and biological evaluation of brain-specific glucosyl thiamine disulfide prodrugs of naproxen.,Eur J Med Chem. 2011; 46:3651-61.

[49] R Gabathuler.Approaches to transport therapeutic drugs across the blood-brain barrier to treat brain diseases Neurobiology of Disease. 2010; 37(1): 48-57.

[50] Banks WA. From blood-brain barrier to blood-brain interface: new opportunities for CNS drug delivery. Nat Rev Drug Discov. 2016; 15:275-92.

[51] WM Pardridge.Drug transport across the blood-brain barrier Journal of Cerebral Blood Flow \& Metabolism. 2012; 32(11):1959-1972. 